\title{
Efecto de la ingesta de dietas con tomate (Lycopersicum esculentum) y tomate de árbol (Cyphomandra betacea (Cav.) Sendtn) en los lípidos sanguíneos de ratas
}

\author{
Effect of dietary intake of tomato \\ (Lycopersicum esculentum) and tree tomato \\ (Cyphomandra betacea (Cav.) Sendtn) \\ on blood lipids in rats
}

\begin{abstract}
Consumption of tomato (Lycopersicon esculentum) and tree tomato (Cyphomandra betacea) is interesting for its nutritional composition and content of antioxidant compounds. This research evaluated the effect of diets intake with tomatoes on blood lipids in rats. First, fatty acids were determined in tomato seeds. Subsequently, rats (Sprague-Dawley, 6 females per group , 2 months old and weight $160 \mathrm{~g}$ ) were fed with basal diet (AIN$93 \mathrm{M})$ supplemented with $10 \%$ tomato and tomato tree at the expense of starch for 5 weeks. Efficiency and diet digestibility were calculated. Triglycerides (plasma), total cholesterol (serum), HDL (plasma) were measured and LDL was calculated. Results showed that seeds of $C$. betacea $7 \%$ had more polyunsaturated fatty acids than L. esculentum. Consumption of diets with both fruits decreased triglycerides and $L D L$ and increased HDL. The results indicate the importance of encouraging the consumption of these fruits as a contribution to the prevention of some chronic diseases.

Key words: tomato, tree tomato, antioxidant compounds, fatty acids, blood lipids.
\end{abstract}

Alexia Torres $P$ Julieta Guinand $Q$.

Departamento de Procesos Biológicos y Bioquímicos. Universidad Simón Bolívar. Caracas-Venezuela.

Dirigir la correspondencia a: Profesora Alexia Torres $P$

Departamento de Procesos Biológicos y Bioquímicos Universidad Simón Bolívar. ZP 1080-A Caracas, Venezuela Teléfono: +582129063111. F-mail.aitorres@ush ve

Este trabajo fue recibido el 9 de Julio de 2013 y aceptado para ser publicado el 1 de Noviembre de 2013.

\section{INTRODUCCIÓN}

La tasa de mortalidad cardiovascular en los países en desarrollo ha presentado un incremento significativo en los últimos años. En Venezuela, constituye la primera causa de muerte en la población, representando $21 \%$ de la mortalidad diagnosticada (1). En América Latina también se evidencia un aumento significativo de dichas enfermedades asociado a cambios en los estilos de vida, aumento demográfico y una transición epidemiológica y nutricional (2).

En un estudio de casos de factores de riesgo sobre estas enfermedades (Interheart) (2), se demostró, que a nivel mundial el incremento en los lípidos sanguíneos, fue el factor más importante, tanto en hombres (49,5\%) como en mujeres (47,1\%). En América Latina, el mayor riesgo se debió a obesidad abdominal (45,8\%). Dentro de algunos parámetros evaluados que tuvieron un efecto protector, se señalan el consumo diario de frutas y/o verduras y el ejercicio.

El informe sobre "Dieta, Nutrición y Prevención de Enfermedades Crónicas" publicado por WHO/FAO el año 2003 (3), reconoció que la evidencia científica asociada a la disminución del riesgo de enfermedades cardiovasculares en las personas que consumen al menos $400 \mathrm{~g}$ de frutas y hortalizas al día era convincente y un elemento probable en la disminución del riesgo de algunos tipos de cánceres. Las frutas y vegetales contienen un amplio rango de compuestos nutritivos como las vitaminas y no nutritivos como la fibra y polifenoles con potencial efecto cardioprotector (4-6).

Se ha renovado el interés por la posibilidad de emplear fibra soluble en el tratamiento de la diabetes, la dislipidemia y la obesidad. Investigadores señalan que el consumo de fibra soluble está relacionado con una disminución tanto del colesterol total como de las lipoproteínas de baja densidad (LDL). Así mismo, las dietas ricas en fibra disminuyen los valores de triglicéridos (TAG) por inhibición de la lipogénesis hepática $(7,8)$.

El tomate (L. esculentum) y sus productos derivados son considerados alimentos saludables por ser bajos en calorías y grasas, libres de colesterol y aportar un adecuado conte- 
nido de fibra, potasio, magnesio y compuestos flavonoides. Adicionalmente, son ricos en vitaminas $A, B_{1}, B_{2}, B_{5}, C, \alpha, \beta$, Y-carotenos, luteína, siendo la principal fuente de licopeno en la dieta (9-11). Las semillas representan el $10 \%$ de la fruta y un $60 \%$ del residuo total siendo consideradas una fuente de proteínas (35\%) y grasas (25\%). En la literatura (6) se ha reportado la importancia del aceite de las semillas por su composición en ácidos grasos insaturados, incluyendo el ácido oleico y los ácidos esenciales linoleico, $y$-linolénico y $\alpha$-linolénico.

Estudios epidemiológicos revelan que un alto consumo de tomate mejora el estatus antioxidante, disminuye la peroxidación de lípidos plasmáticos y mejora la defensa de las LDL frente al ataque del oxígeno singlete $(12,13)$. Por su parte, el tomate de árbol (C.betacea) es apreciado por sus cualidades (vitaminas $A, B$ y $C$ ), minerales (calcio, fósforo, hierro y potasio), fibra soluble e insoluble y por ser fuente de compuestos con potencial antioxidante y cardioprotector como el ácido Y-aminobutírico, el licopeno, los polifenoles y las antocianinas, atribuyéndole propiedades de reducción del colesterol $(14,15)$.

En vista de la escasa literatura (16) referente a la composición de ácidos grasos de las semillas de tomate de árbol, se planteó en esta investigación comparar el contenido de ácidos grasos de las semillas de L. esculentum y C.betacea y evaluar el efecto de la ingesta de ambos frutos en la dieta sobre los lípidos sanguíneos en ratas.

\section{MATERIALES Y MÉTODO} Muestras

Para la obtención de las semillas y la elaboración de las dietas se emplearon $6 \mathrm{~kg}$ de cada tipo de tomate ( $L$. esculentum y C. betacea) adquiridas de un productor local en el Jarillo, Edo. Aragua, Venezuela.

Los frutos fueron lavados y sumergidos en agua con hipoclorito de sodio 50 ppm/15 min. para su desinfección. Posteriormente se dividieron en dos subgrupos. Del primer subgrupo, se tomaron $0,5 \mathrm{~kg}$ de cada variedad de tomate ( $L$. esculentum y $C$. betacea) y se extrajeron las semillas las cuales fueron secadas en horno de convección forzada a $45^{\circ} \mathrm{C} / 24$ horas y molidas hasta tamaño de partícula de 40 mesh, para determinación de ácidos grasos.

El segundo subgrupo estuvo formado por $2,5 \mathrm{~kg}$ de $C$. betacea, los cuales fueron pelados (como es usualmente procesado) y rebanados y $2,5 \mathrm{~kg}$ de $L$. esculentum, los cuales sólo se rebanaron. Los frutos fueron secados en horno de convección forzada a $45^{\circ} \mathrm{C} / 24$ horas y molidos hasta tamaño de partícula de 40 mesh, para ser incorporados en la dieta de los animales de experimentación.

Determinación de humedad, grasa total y ácidos grasos en semillas de $L$. esculentum y $C$. betacea

La humedad se determinó empleando secado en estufa de vacío (Labconco, Kansas City, MO, USA), a presión de 24 $\mathrm{mm} \mathrm{Hg} / 65^{\circ} \mathrm{C}$ hasta alcanzar peso constante (17) (método AOAC, 940.25). La grasa total fue extraída empleando el método Soxhlet utilizando hexano como solvente (17) (método AOAC, 940.12).

Los ácidos grasos (AG) fueron extraídos de acuerdo al método de Folch y col. (18). La composición de ácidos grasos fue determinada por cromatografía gas/líquido después de la transesterificación de los AG con una mezcla metanol/tolueno/ ácido sulfúrico (86:10:4) e incubados por $90 \mathrm{~min}$ a $80^{\circ} \mathrm{C}(19)$. Los metil ésteres de los ácidos grasos fueron determinados después de su separación empleando un cromatógrafo de gases (marca HP, modelo 6890 Plus GC, versión A.03.07), con una columna capilar recubierta interiormente de una película de cianopropil 0, $2 \mu \mathrm{m}$ con una longitud de $100 \mathrm{~m}$ y d.i de $0,25 \mathrm{~mm}$ (HP-88). El resultado se expresó como g/100g de ácidos grasos.

\section{Diseño de experimento}

Se trabajó con 18 ratas hembras de la raza SpragueDawley, de dos meses de edad (peso aproximado de $160 \mathrm{~g}$ ) las cuales fueron mantenidas en grupos de 6 por tratamiento, en jaulas de experimentación individuales. Se les administraron los siguientes tipos de dieta: control o basal (AIN-93M) y agua ad libitum (DB), dietas (AIN-93M) suplementadas con $10 \%$ de tomate y agua ad libitum (DT), y con $10 \%$ de tomate de árbol y agua ad libitum (DTAR). Los tomates fueron incorporados a expensas del almidón siguiendo protocolo descrito en la literatura (11). Las dietas suplementadas con tomate y tomate de árbol fueron formuladas para proveer la misma cantidad de energía, proteína, grasa, fibra, vitaminas y minerales que la dieta control (AIN-93M) (DB), de acuerdo a estudios de composición reportados para dichos frutos $(20,21)$ y se les calculó teóricamente el contenido de proteínas, carbohidratos, lípidos y energía. Los animales fueron acondicionados en el ambiente experimental por un período de tres días con dieta basal preparada de acuerdo a las recomendaciones reportadas por la literatura (22). Las ratas fueron mantenidas en períodos alternos de 12 horas de luz y 12 horas de oscuridad, por un período de 5 semanas. A lo largo del experimento se registró el peso corporal y el consumo de alimento de forma inter diaria. Durante las últimas $48 \mathrm{~h}$ del período experimental se recolectaron las heces para determinar el peso húmedo y posteriormente el peso seco.

Los ingredientes empleados en la preparación de la dieta, así como el aporte de proteínas, carbohidratos, lípidos y energía de las mismas, se presentan en la tabla 1.

Al finalizar el estudio, las ratas fueron anestesiadas con éter etílico y posteriormente se obtuvo una muestra de sangre por punción cardíaca, de la cual se separó una parte para analizar el suero (centrifugación a $2500 \mathrm{rpm}$ por $15 \mathrm{~min}$ a $4^{\circ} \mathrm{C}$ y conservación a $-20^{\circ} \mathrm{C}$ ) y otra se colocó en tubos con heparina para luego obtener el plasma (conservación a $-20^{\circ} \mathrm{C}$ ).

En esta investigación se siguieron los procedimientos y principios establecidos por la Comisión de Bioética de la Universidad Simón Bolívar, Caracas-Venezuela.

\section{Cálculo de la utilización de la dieta}

La eficiencia alimentaria se calculó de acuerdo a la siguiente fórmula:

Eficiencia alimentaria $(\%)=$

(ganancia peso/consumo alimento) ${ }^{*} 100$

Cálculo de la digestibilidad de la dieta

Para el cálculo de la digestibilidad de la dieta se determinó la humedad de las heces (método AOAC, 940.25) (17).

La digestibilidad se calculó aplicando la siguiente fórmula: Digestibilidad de la dieta $(\%)=$

(consumo (base seca)-peso heces (base seca))/consumo)*100

Determinaciones bioquímicas en suero y plasma

Para la determinación de triglicéridos (TAG) en plasma se utilizó el kit enzimático método Trinder, Heiga, Ref 880-L (23). La determinación de colesterol total (CT) en muestras de suero se hizo empleando el kit enzimático, método Trinder, Heiga Ref 450-L y el colesterol HDL en muestras de suero por el método de fosfotungstato de sodio/cloruro de magnesio 
TABLA 1

Composición de las dietas utilizadas en el diseño de experimentos.

\begin{tabular}{lccc}
\hline Ingredientes (g/100g) & DT & \\
& & 12,74 & 12,74 \\
Aislado de soya & 14,00 & 10,00 & 10,00 \\
Sacarosa & 10,00 & 4,09 & 4,09 \\
Celulosa & 5,00 & 54,53 & 54,53 \\
Almidón maíz & 62,21 & 0,03 & 0,03 \\
Cisteína & 0,03 & 0,25 & 0,25 \\
Bitartrato de colina & 0,25 & 0,002 \\
t-butil hidroquinona (TBHQ) & 0,002 & 3,002 & 1,00 \\
Mezcla minerales & 3,50 & 3,50 & 3,85 \\
Mezcla vitaminas & 1,00 & 1,00 & 0 \\
Aceite soya & 4,00 & 3,85 & 10,00 \\
Tomate & 0,00 & 10,00 & 100,00 \\
Tomate de árbol & 0,00 & 0,00 & 100,00 \\
Total & 100,00 & \\
\hline
\end{tabular}

DB (dieta basal y consumo agua); DT (dieta con tomate y consumo de agua); DTAR (dieta con tomate de árbol y consumo de agua).

Composición de las dietas: Proteínas (\%) : DB 11,30, DT 11,60 y DTAR 11,72; Carbohidratos (\%): DB 66,08, DT 66,82 y DTAR 63,60;

Lípidos (\%): DB 4,47, DT 4,34 y DTAR 4,42; Energía (Kcal/100g): DB 316, DT 316, DTAR 307

TABLA 2

Perfil de ácidos grasos de las semillas de tomate normal y de tomate de árbol

\begin{tabular}{|c|c|c|c|}
\hline $\begin{array}{c}\text { Ácidos grasos } \\
\text { (g/100 g total grasa) }\end{array}$ & $\begin{array}{l}\text { Semillas de } \\
\text { tomate }\end{array}$ & $\begin{array}{c}\text { Semillas de } \\
\text { tomate de árbol }\end{array}$ & Nombre común \\
\hline $12: 0$ & $0,01 \pm 0,00$ & ND & Ácido laúrico \\
\hline $14: 0$ & $0,13 \pm 0,01$ & $0,05 \pm 0,00$ & Ácido mirístico \\
\hline $15: 0$ & $0,04 \pm 0,02$ & $0,01 \pm 0,00$ & Ácido pentadecanoico \\
\hline $16: 0$ & $13,15 \pm 0,01$ & $8,93 \pm 0,04$ & Ácido palmítico \\
\hline $16: 1 n-7$ & $0,24 \pm 0,04$ & $0,43 \pm 0,02$ & Ácido palmitoleico \\
\hline $17: 0$ & $0,07 \pm 0,02$ & $0,07 \pm 0,01$ & Ácido heptadecanoico \\
\hline $17: 1$ & $0,05 \pm 0,00$ & $0,06 \pm 0,02$ & Ácido heptadecenoico \\
\hline 18:0 & $5,12 \pm 0,02$ & $3,54 \pm 0,02$ & Ácido esteárico \\
\hline $18: 1 n-9$ & $20,94 \pm 0,02$ & $19,75 \pm 0,00$ & Ácido oleico \\
\hline $18: 2 n-6$ & $56,48 \pm 0,02$ & $63,36 \pm 0,00$ & Ácido linoleico \\
\hline $18: 3 n-6$ & $0,39 \pm 0,01$ & $0,42 \pm 0,01$ & Ácido g-linolénico \\
\hline $18: 3 n-3$ & $2,89 \pm 0,01$ & $3,28 \pm 0,03$ & Ácido a-linolénico \\
\hline 20:0 & $0,49 \pm 0,00$ & $0,47 \pm 0,00$ & Ácido eicosanoico \\
\hline $20: 1 n-6$ & $0,11 \pm 0,00$ & $0,11 \pm 0,00$ & Ácido eicosaenoico \\
\hline $20: 3 n-6$ & $0,16 \pm 0,00$ & $0,29 \pm 0,00$ & Ácido dihomo-g -linolénico \\
\hline $21: 0$ & $0,06 \pm 0,00$ & $0,07 \pm 0,00$ & Ácido heneicosanoico \\
\hline $22: 1$ & ND & $0,02 \pm 0,00$ & Ácido docosaenoico \\
\hline $22: 2$ n 6 & ND & $0,02 \pm 0,01$ & Ácido docosadienoico \\
\hline $23: 0$ & $0,03 \pm 0,00$ & $0,05 \pm 0,00$ & Ácido tricosanoico \\
\hline $24: 0$ & $0,12 \pm 0,00$ & $0,38 \pm 0,00$ & Ácido lignocérico \\
\hline Total AGS & 19,22 & 13,57 & \\
\hline Total AGMI & 21,34 & 20,37 & \\
\hline Total AGPI & 59,92 & 66,95 & \\
\hline Total AGI & 81,26 & 87,32 & \\
\hline
\end{tabular}

La tabla muestra la media y la desviación estándar de $\mathrm{n}=2$ muestras. 
(kit diagnóstico Heiga Ref 460) (24). El colesterol LDL fue calculado (25).

\section{Análisis estadístico}

Las variables estudiadas se reportaron como media y desviación estándar. Para todos los análisis se estableció un nivel de significancia del 0,05 y se utilizó el programa estadístico Statgraphics Centurion XV para evaluar el efecto de las dietas sobre las variables en estudio. Los resultados fueron analizados por una prueba de comparación múltiple y una vez verificados los supuestos de normalidad, independencia y homogeneidad de la varianza, se realizó el contraste de medias por el método de rangos múltiples de Duncan.

\section{RESULTADOS Y DISCUSIÓN}

Análisis del perfil de ácidos grasos de las semillas de los tomates

Los resultados del análisis del perfil de ácidos grasos de las semillas de ambos frutos se presentan en la tabla 2 . El valor obtenido de grasa de las semillas fue $23,5 \%$ y $24,32 \%$ para el tomate y el tomate de árbol, valor superior al reportado por la bibliografía (16). Con respecto a los ácidos grasos poliinsaturados, se evidencia $7 \%$ más en el tomate de árbol y $6 \%$ menos de ácidos grasos saturados, que en el tomate. Estos resultados coinciden con los reportados en la literatura tanto para el tomate como el tomate de árbol $(6,16)$. Los autores concluyen que el aceite de las semillas de ambos frutos se considera rico en ácido linoleico, superando en ambos casos el contenido del mismo en aceites de soya, maíz, sésamo, girasol y algodón.

Algunos estudios $(26,27)$ citan la función bien establecida del ácido linoleico para bajar los niveles de colesterol en sangre. De acuerdo a la reunión de expertos sobre grasas (27), el consumo de ácido linoleico hasta en 3,5\% de la ingesta total de grasa, forma parte de una dieta saludable que contribuye a largo plazo a la salud, bajando las concentraciones de colesterol total y LDL colesterol. Estos investigadores $(26,27)$, además reconocen la evidencia clínica y epidemiológica que prueba el valor de las grasas $\mathrm{w}-3$.

El aceite de las semillas analizadas presenta una proporción de ácidos grasos mono (21,34 y 20,37\% para tomate y tomate de árbol respectivamente) y poli-insaturados $(59,92$ y $66,95 \%$ para tomate y tomate de árbol respectivamente) semejantes a la reportada en semillas de uva, de algodón y mostaza $(28,29)$.

Efecto producido por las dietas en los lípidos sanguíneos de las ratas

Previamente al estudio del efecto del consumo de dietas con tomate sobre los lípidos sanguíneos de las ratas, se determinó en los animales de experimentación el consumo promedio, la eficiencia y digestibilidad del alimento (tablas 3 y 4). Se evidenció un incremento de peso de los animales y una variación del consumo promedio de alimento entre 470

\section{TABLA 3}

Datos de ganancia de peso, consumo total, eficiencia alimentaria de tres grupos de ratas Sprague-Dawley alimentadas con una dieta basal, dieta con tomate y tomate de árbol.

\begin{tabular}{|c|c|c|c|}
\hline \multirow[t]{2}{*}{ Parámetro } & \multicolumn{3}{|c|}{ Dietas } \\
\hline & DB & DT & DTAR \\
\hline Ganancia de peso (g) & $63,38 \pm 12,82^{\mathrm{a}}$ & $63,40 \pm 7,79^{a}$ & $71,00 \pm 5,94^{a}$ \\
\hline Consumo total de alimento (g) & $470,18 \pm 23,75^{a}$ & $476,62 \pm 32,62^{a}$ & $475,34 \pm 54,20^{a}$ \\
\hline Eficiencia alimentaria (\%) & $13,46 \pm 2,43^{a}$ & $13,27 \pm 0,95^{a}$ & $14,33 \pm 1,51^{\mathrm{a}}$ \\
\hline
\end{tabular}

\section{TABLA 4}

Datos de consumo de alimento, peso heces (húmedas y secas), digestibilidad del alimento de ratas Sprague-Dawley alimentadas con una dieta basal, dieta con tomate y tomate de árbol.

\begin{tabular}{|c|c|c|c|}
\hline \multirow[t]{2}{*}{ Parámetro } & \multicolumn{3}{|c|}{ Dietas } \\
\hline & DB & DT & DTAR \\
\hline Consumo alimento $(g / 2 d)^{1}$ & $35,90 \pm 2,53^{a}$ & $39,40 \pm 7,49^{a}$ & $35,44 \pm 4,11^{a}$ \\
\hline Heces húmedas $(g / 2 d)^{1}$ & $2,74 \pm 0,77^{a}$ & $3,22 \pm 1,36^{a}$ & $3,90 \pm 0,57^{a}$ \\
\hline \multicolumn{4}{|l|}{ Heces secas $(g / 2 d)^{1}$} \\
\hline & $2,03 \pm 0,44^{a}$ & $2,19 \pm 0,84^{a}$ & $2,43 \pm 0,30^{\mathrm{a}}$ \\
\hline Digestibilidad del alimento (\%) & $93,10 \pm 1,78^{a}$ & $92,52 \pm 3,35^{\mathrm{a}}$ & $89,99 \pm 1,74^{a}$ \\
\hline
\end{tabular}


a $476 \mathrm{~g}$ durante el tiempo del estudio. No hubo diferencia $(p>0,05)$ en relación a la eficiencia alimentaria. Los resultados obtenidos ponen de manifiesto que los animales toleraron bien el sabor y aroma de los ingredientes de tomate.

Los valores de los parámetros bioquímicos evaluados en los animales de experimentación se observan en la tabla 5 . Para la variable TAG, el contraste de la respuesta entre el grupo que consumió DB y los animales que consumieron tanto $L$. esculentum como $C$. betacea, permitió detectar una reducción estadísticamente significativa $(p<0,05)$, de $33 \%$ y $42 \%$ para DT y DTAR respectivamente.

Investigaciones previas realizadas al fruto de tomate y tomate de árbol (15) y datos reportados por otros investigadores $(30,31)$, evidencian un contenido de pectina entre $1 \mathrm{y}$ $2,5 \%$, así como un aporte de fibra dietaria $(0,27 \%$ soluble y $0,84 \%$ insoluble para tomate, así como $1,89 \%$ soluble y 2,21 $\%$ insoluble en tomate de árbol) lo cual pudiera justificar el efecto observado en este estudio en la disminución de los TAG.

Investigadores (32) han resaltado el hecho de que el tomate (L. esculentum) es uno de los cultivos más conocidos y contiene compuestos beneficiosos que ayudan a mejorar las anormalidades del metabolismo lipídico, a pesar de que no está claro el mecanismo involucrado. En estudios con licopeno de tomate (32), se evaluó que la respuesta no era atribuida al caroteno sino a la presencia del ácido 9-oxo-octadecadienóico (9-oxo-ODA), la cual es una oxilipina presente en plantas, que actúa sobre el receptor activado por proliferadores de peroxisoma (aPPARa).Se señala que la activación de este receptor aumenta la b-oxidación lo cual resulta en una disminución de los triglicéridos en plasma e hígado. En el fruto de C. betacea sería recomendable evaluar la presencia de este compuesto, para poder establecer una comparación.

Con respecto al $\mathrm{CT}$, no se observó un efecto significativo $(p>0,05)$ de las dietas, lo cual coincide con lo reportado en la literatura (33) donde se resalta el hecho de que las ratas tienen un sistema muy eficiente para mantener la homeostasis del colesterol sanguíneo.

Al relacionar la variable $\mathrm{CT}$ con los TAG se obtuvo que los grupos que consumieron dietas con tomate y tomate de árbol, presentaron un mayor valor estadísticamente significativo $(p<0,05)$ en relación al consumo de DB y similar al reportado en la literatura para animales alimentados con aceite de soya
(34). Este resultado es el reflejo del efecto de la dieta sobre la disminución de los triglicéridos.

Las concentraciones del colesterol HDL aumentaron en promedio $14 \%$ como producto de la ingesta de los frutos (L.esculentum y C.betacea) en relación a la dieta basal, no siendo estadísticamente significativo $(p>0,05)$. Estos resultados se soportan con lo reportado en la literatura (35), donde se observó una relación inversamente proporcional entre el aumento del colesterol HDL y la disminución de los TAG.

Para la relación $\mathrm{CT} / \mathrm{HDL}$, hay un efecto significativo $(p<0,05)$ de la ingesta de productos de tomate sobre la disminución del índice respectivo similar a lo reportado con animales alimentados con aceite de soya (34), en concordancia con el efecto que tuvieron las dietas con los frutos, sobre la variable HDL.

De los resultados del colesterol LDL, hubo una disminución del $22 \%$ para la dieta de tomate y de $33 \%$ para la dieta de tomate de árbol no siendo estadísticamente significativo en relación a la dieta basal. Estos resultados pueden ser atribuidos a la presencia de fibra dietaria en ambos frutos determinadas en estudios anteriores $(15,31)$, así como al aporte de ácidos grasos poliinsaturados provenientes de las semillas de los tomates y del aceite de soya utilizado en la formulación de las dietas. Es bien conocido el hecho que demuestra que la fibra soluble reduce los lípidos sanguíneos por sus propiedades viscosas que atrapan los ácidos biliares (36).

\section{CONCLUSIONES}

En las semillas de tomate de árbol se encontró un 7\% más de ácidos grasos poliinsaturados y un $6 \%$ menos de ácidos grasos saturados con respecto al tomate. En las condiciones de experimentación "in vivo", se pudo comprobar que el consumo de una dieta con L.esculentum y C.betacea tuvo un efecto significativo sobre la disminución de los TAG. También se evidenció un aumento del colesterol HDL y una disminución del colesterol LDL para ambas dietas. Los resultados señalan la importancia de incentivar el consumo de ambos frutos como una contribución a la prevención de enfermedades.

RESUMEN

El consumo de tomate (Lycopersicum esculentum) y tomate de árbol (Cyphomandra betacea) resulta de interés

\section{TABLA 5}

Datos de los indicadores bioquímicos de ratas Sprague-Dawley alimentadas con una dieta basal, dieta con tomate y tomate de árbol.

Parámetro

Triglicéridos plasma TAG $(\mathrm{mg} / \mathrm{dl})$

Colesterol total suero $\mathrm{COL}(\mathrm{mg} / \mathrm{dl})$

$\mathrm{COL} / \mathrm{TAG}$

HDL-colesterol plasma $(\mathrm{mg} / \mathrm{dl})$

$\mathrm{COL} / \mathrm{HDL}$

LDL-colesterol ( $\mathrm{mg} / \mathrm{dl})$

Índice LDL/HDL
DB

$80,79 \pm 9,93 b$
$83,67 \pm 6,19 a$
$1,05 \pm 0,18 a$
$64,23 \pm 9,42 a$
$1,41 \pm 0,22 b$
$13,15 \pm 4,96 a$
$0,20 \pm 0,09 a$

Dieta

DT

$53,73 \pm 8,96 a$
$85,88 \pm 13,07 a$
$1,62 \pm 0,50 b$
$74,28 \pm 8,28 a$
$1,19 \pm 0,14 a$
$10,25 \pm 2,88 a$
$0,15 \pm 0,05 a$

$53,73 \pm 8,96 a$

$47,26 \pm 16,74 a$

$82,91 \pm 6,26 a$

$2,09 \pm 0,51 \mathrm{c}$

$72,60 \pm 12,88 a$

$1,19 \pm 0,16 a$

$8,84 \pm 3,99 a$

$0,13 \pm 0,06 a$

DB (dieta basal y consumo agua); DT (dieta con tomate y consumo de agua); DTAR (dieta con tomate de árbol y consumo de agua).

La tabla muestra la media y la desviación estándar de $n=6$ animales por grupo. Medias en una misma fila con letras distintas indican diferencia estadística de acuerdo a la prueba de rangos múltiples de Duncan $(a=0,05)$. 
por su composición nutricional y contenido de compuestos antioxidantes. Esta investigación evaluó el efecto de la ingesta de dietas con tomates en los lípidos sanguíneos de ratas. Primero, se determinaron los ácidos grasos en semillas de tomates. Posteriormente, a ratas (Sprague-Dawley, 6 hembras por grupo, 2 meses de edad y peso $160 \mathrm{~g}$ ), se les suministró dieta basal (AIN-93M), dietas suplementadas con 10\% de tomate y de tomate de árbol a expensas del almidón, durante 5 semanas. Se calculó la eficiencia y digestibilidad de la dieta. Se midieron los triglicéridos (plasma), colesterol total (suero), HDL (plasma) y LDL calculado. Resultados indicaron 7\% más de ácidos grasos poliinsaturados en semillas de $C$. betacea respecto a $L$. esculentum. El consumo de dietas con ambos frutos disminuyó los triglicéridos y LDL y aumentó el HDL. Los resultados señalan la importancia de incentivar el consumo de ambos frutos como una contribución a la prevención de algunas enfermedades crónicas.

Palabras clave: tomate, tomate de árbol, compuestos antioxidantes, ácidos grasos, lípidos sanguíneos.

\section{BIBLIOGRAFÍA}

1. Gobierno Bolivariano de Venezuela. Ministerio Para el Poder Popular de la Salud. Anuario de Mortalidad 2010. Caracas-Venezuela, 2012.

2. Lanas F. Toro V. Cortés R. Sánchez A. Interheart, Un Estudio de Casos y Controles sobre Factores de Riesgo de Infarto del Miocardio en el Mundo y América Latina. Médicas UIS. 2008; 21:176-82.

3. WHO/FAO. Diet Nutrition and The Prevention of Chronic Disease. WHO technical report series 916, Geneva, 2003.

4. Hollman PCH. Arts I C. Flavonols, flavones and flavanols: Nature, occurrence and dietary burden. J Sci Food Agric. 2000; 80:1081-93.

5. Naczk M. Shahidi F. Phenolics in cereal, fruit and vegetables: Ocurrence extraction and analysis. J Pharm and Biom Anal. 2006; 41:1523-42.

6. Schieber A. Stintzing F C. Carle R. By-products of plant food processing as a source of functional compounds recent developments. Trends in Food Sci Technol. 2001; 12: 401-13.

7. Fernandez ML. Soluble fiber and nondigestible carbohydrate effects on plasma lipids and cardiovascular risk. Current Opinion Lipidol. 2001; 12:35- 40.

8. Venkatesan N. Devaraj S N. Devaraj H. Increased binding of $L D L$ and VLDL to apo $B, E$ receptor of hepatic plasma membrane of rat treated with fiber. Eur J Nutr 2003; 42 . 262-71.

9. Pineda D. Capacidad antioxidante y potencial sinergismo entre los principales constituyentes antioxidantes de algunos alimentos. Rev. Cubana Alim Nutr. 1999; 13(2):104-11.

10. Palomo I. Moore-Carrasco R. Carrasco G. Villalobos P. Guzmán L. El consumo de tomates previene el desarrollo de enfermedades cardiovasculares y cáncer: antecedentes epidemiológicos y mecanismos de acción. IDESIA 2010; 28 (3): 121-9.

11. Alshatwi AA. Al Obaaid MA. Al Sedairy SA. Al-Assaf AH. Zhang JJ. Lei KY. Tomato powder is more protective than lycopene supplement against lipid peroxidation in rats. Nutr Res 2010; 30:66-73.

12. Riso P. Pinder A. Santangelo A. Porrini M. Does tomato consumption effectively increase the resistance of lymphocyte DNA to oxidative damage? Am J Clin Nutr 1999; 69(4):712-718.

13. Johnston C. Functional Foods as Modifiers of Cardiovascular
Disease. Am J Lifestyle Med. 2009; 3 (1 Suppl): 39S-43S.

14. Lister C. Morrison S. Kerkhofs N. Wright K. The nutritional composition and health benefits of New Zealand tamarillos. Crop \& Food Research Confidential Report No. 1281. Edited by New Zealand Institute forCrop \& Food Research Limited. New Zealand, 2005, p 29.

15. Torres A. Caracterización física, química y compuestos bioactivos de pulpa madura de tomate de árbol (Cyphomandra betacea) (Cav.) Sendtn. Arch Lat Nutr. 2012; 4(62):381-8.

16. Belén-Camacho DR. Sánchez E. García D. Moreno-Alvarez $J M J$. Linares $O$. Características fisicoquímicas y composición en ácidos grasos del aceite extraído de semillas de tomate de árbol (Cyphomandra betacea Sendt) variedades roja y amarilla. Grasas aceites 2004; 55(4):428-33.

17. AOAC. Official Methods of Analysis 18th Edition. Dr. William Horwitz, Editor Dr. George W. Latimer, Jr., Assistant Editor. Pub AOAC International, Maryland, USA. 2005.

18. Folch J. Lees M. Sloane GH. A simple method for the isolation and purification of total lipids from animal tissue. J Biol Chem. 1957; 228:497-509.

19. Lepage G. Roy C. Direct transesterification of all classes of lipids in a one-step reaction. J. Lipid Res. 1986; 27:114-20.

20. Mendoza H. Composición nutricional, características sensoriales y vida de anaquel de tomate (Lycopersicum esculentum) cultivado en sustrato orgánico, hidropónico y convencional. Tesis grado. Universidad de San Carlos de Guatemala. Facultad de Ciencias Químicas y Farmacia. Guatemala. 2005.

21. FAO. Tomate de árbol. Ficha Técnica. 2006. Disponible en: http://www.fao.org/inpho/content/documents/vlibrary/ ae620s/Pfrescos/TOMATEDEARBOL.HTM. consultada 20-02-2013

22. Reeves PG. Nielsen FM. Fahey GC. AIN-93 purified diets for laboratory rodents: final report of the American Institute of Nutrition ad hoc writing committee on the reformulation of the AIN-76A rodent diet. J Nutr. 1993; 123:1939-51.

23. Trinder P. Enzymatic methods of tryglicerides. Am Clin Biochem. 1969; 6:24-7.

24. Allain C. Poon L. Chen C. Richmond W. Fu P. Enzymatic determination of the total serum cholesterol. Clin Chem.1974; 20:470-5.

25. Friedewald W. Levy R. Fredickson S. Estimation of the concentration of low density lipoprotein cholesterol in plasma without use the preparative ultracentrifugue. Clin Chem.1972; 18:469-75

26. Fundación del Consejo Internacional de Información Alimentaria. Hoja de datos sobre ácidos grasos omega 6 y salud. Octubre 2010. Disponible:http://www.foodinsight. org/enespanol/Resources/. Consultada 20-05-2013

27. FAO-FINUT. Grasas y ácidos grasos en nutrición humana. 2008 Consulta de expertos. 10-14 Nov 2008, Ginebra. Estudio FAO Alimentación y Nutrición 91, 2012.pp: 17.

28. Navas $P$ B. Caracterización fisicoquímica del aceite de semillas de uva extraído con solvente en frío. Rev Fac Agron (LUZ) 2010; 27:270-88.

29. Norma del Codex para aceites vegetales especificados. Codex Stan 210-1999.

30. Devia J.R. Proceso para producir pectinas cítricas. Rev Universidad EAFIT. 2003; 129: 21-30.

31. Pak D N. Fibra dietética en verduras cultivadas en Chile. Arch Latinoam Nutr. 2000; 50(1):97-101.

32. Kim Y. Hirai S. Takahashi H. Goto T. Ohyane C. Tsugane T. Konishi C. Fujii T. Inai S. lijima Y. Aoki K. Shibata D. Takahashi N. Kawada T. 9-oxo-10(E,12(E)-octadecadienoic acid 
derived from tomato is a potent PPAR agonist to decrease trygliceride accumulation in mouse primery hepatocytes. Mol Nutr Food Res. 2010; 54: 1-9.

33. Soler TW. Rodríguez DC. Angarita V B. Corredor P C. Efecto de la dieta sobre lípidos de la sangre y el hígado de ratas. Salud Uninorte 1986; 3 (1): 19.29.

34. Almeida M E. Quieroz J H. Costa N. Matta S. Lipídeos séricos e morfologia hepática de ratos alimentados com diferentes fontes lipídicas (óleo de soja, gordura de peixe e porco, margarina e manteiga) Rev Nutr Campinas 2011; 24(1):143-52.

35. Lamarche B. Rashid S. Lewis GF. HDL metabolism in hypertriglyceridemic states. An overview. Clin Chim Acta 1999; 286: 145-61.

36. Swain JF, Rouse IL, Curley CB, Sacks FM. Comparison of the effects of oat bran and low-fiber wheat on serum lipoprotein levels and blood pressure. N Engl J Med.1990;322 (3):147-52. 